Relations industrielles

Industrial Relations

\title{
Personnal Management. A Human Resource System Approach, by Elmer H. Burack et Robert D. Smith, New York, West Publishing C., N.Y., 1977, 513 pp.
}

\section{Laurent Bélanger}

Volume 32, numéro 1, 1977

URI : https://id.erudit.org/iderudit/028773ar

DOI : https://doi.org/10.7202/028773ar

Aller au sommaire du numéro

Éditeur(s)

Département des relations industrielles de l'Université Laval

ISSN

0034-379X (imprimé)

1703-8138 (numérique)

Découvrir la revue

Citer ce compte rendu

Bélanger, L. (1977). Compte rendu de [Personnal Management. A Human Resource System Approach, by Elmer H. Burack et Robert D. Smith, New York, West Publishing C., N.Y., 1977, 513 pp.] Relations industrielles / Industrial Relations, 32(1), 146-146. https://doi.org/10.7202/028773ar

Tous droits réservés @ Département des relations industrielles de l'Université Laval, 1977
Ce document est protégé par la loi sur le droit d'auteur. L'utilisation des services d’Érudit (y compris la reproduction) est assujettie à sa politique d'utilisation que vous pouvez consulter en ligne.

https://apropos.erudit.org/fr/usagers/politique-dutilisation/ 
se that induces dissatisfaction, but the linking of arbitration to other procedures such as limiting access to arbitration to union registration in Great Britain in the 1971 Industrial Relations Act». Quant à nous, cette observation n'a pu nous faire oublier les nombreuses remarques faites à plusieurs reprises dans l'ouvrage du genre «increasing dissillusion about the process in Jamaica and Australia», "failure» du système australien, «undesirable but sometimes necessary evil», et autres. Enfin, il y a lieu de noter que les auteurs insistent particulièrement sur le fait que dans les pays où il $\mathrm{y}$ a «free collective bargaining», l'arbitrage obligatoire des différends ne peut être viable que si toutes les parties acceptent de vivre avec un tel système.

Compulsory Arbitration est, en définitive, un ouvrage qui ne peut que soulever un immense intérêt. Nous terminons par cette remarque fort lucide contenue au dernier paragraphe du volume: "Little can be gained at this point from arguing whether compulsory arbitration is an appropriate part of industrial relations. Rather, the questions for each country are: When should compulsory arbitration be adopted, and what are the likely implications of alternatives structures? Some of the answers may be found in the experience recounted here. The remainder will come from experiences that lie ahead».

\section{Rodrigue BLOUIN}

\section{Université Laval}

Personnal Management. A Human Resource System Approach, by Elmer $\mathrm{H}$. Burack et Robert D. Smith, New York, West Publishing C., N.Y., 1977, 513 pp.

Cet ouvrage, comme le titre l'indique, couvre tout le domaine de la gestion des ressources humaines, et cela dans un contexte économique et juridique toujours changeant, ce qui a incité les auteurs à organiser les connaissances en matière de gestion des ressources humaines en prenant comme point de départ les changements majeurs qui affectent l'entreprise et l'utilisation de ses différentes catégories de personnel. Ceci explique la présence de chapitres nouveaux qui traitent de l'insertion des jeunes au marché du travail, de la gestion du changement, de la gestion des carrières, des nouvelles législations touchant les minorités et l'emploi toujours croissant d'une main-d'œuvre féminine.

L'ouvrage comprend dix-sept chapitres regroupés sous six sections. La présenta- tion de chaque thème suit de près l'enchaînement logique des activités de base inhérentes à la gestion des ressources humaines à savoir l'acquisition, la conservation, le développement et le contrôle des ressources humaines.

Cet ouvrage excelle surtout par le traitement approfondi qu'il accorde à chaque thème, traitement supporté par une présentation de modèles et de techniques que je qualifierais de sophistiqués, un peu à cause du peu de connaissances que j'en ai.

Le volume se distingue également des autres par la pédagogie utilisée. Chaque chapitre s'ouvre par une liste d'objectifs d'apprentissage et une présentation des concepts-clefs dont on retrouve la définition dans un glossaire qui apparaît à la fin. Chaque chapitre est également suivi d'une liste de questions et d'une abondante bibliographie. Après en avoir fait une lecture rapide. je dois constater que l'ouvrage cherche à mettre à la disposition des lecteurs une somme de connaissances qu'on retrouve dans des revues spécialisées et pourtant peu accessibles à la majorité des praticiens. Donc, l'ouvrage serait utile à quelqu'un qui possède déjà une formation initiale dans le domaine ou quelques années d'expérience et qui veut ajouter un peu plus de "professionalisme" dans ce secteur.

Laurent BÉLANGER

Université Laval

Cazeneuve, Jean (sous la dir. de), La sociologie et les Sciences de la Société, Paris, C.E.P.L., 1975, 540 p.

La formule du dictionnaire encyclopédique semble connaître un certain succès, à voir le nombre de publications de ce type sur le marché. L'ouvrage, publié sous la direction de $M$. Jean Cazeneuve, est le deuxième portant sur la sociologie (cf. Cazeneuve J. et Victoroff D. (éd.), $L a$ Sociologie, Paris, C.E.P.L., 1970 et Gérard et Cie, 1977). Inutile de préciser que les répétitions sont nombreuses; toutefois les deux ouvrages sont suffisamment différents pour obliger le lecteur intéressé à se les procurer tous les deux - ce qui n'est pas pour déplaire à l'éditeur - - . On retrouve donc près de 300 mots, expressions et noms d'auteur dont la définition et la présentation constituent la partie $«$ dictionnaire $»$ de l'ouvrage et dix articles qui abordent autant d'aspects de la démarche sociologique. La rédaction de ces articles a été confiée à des chercheurs de longue expérience et ont, dans la plupart des cas, une valeur pédago- 